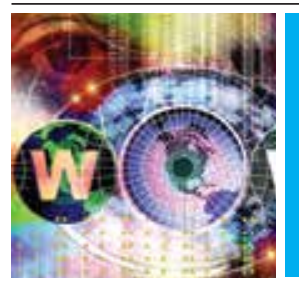

Research Article

\title{
Image Segmentation Using Marker-Controlled Watershed Transformation and Morphology
}

\author{
Hafsa Shabbir, Hajira Bibi, Sania Aamir, Mrs Shazia Saqib and Ms. Noor Afshan \\ fa14-bscs-077@lgu.edu.pk, fa14-bscs-078@lgu.edu.pk,muhammadshan9286@gmail.com \\ shaziasaqib@lgu.edu.pk,noor.afshan@lgu.edu.pk \\ Lahore Garrison University, Lahore, Pakistan.
}

\begin{abstract}
:
The watershed segmentation methods are essential methods, to be considered for quick results in image handling and analysis. However, the main problem arises in produced image because it causes excess segmentation and noise. This research is conducted to improve this presented algorithm based on the mathematical morphology and filters to minimize flaws mentioned in that paper. Objective of this research is to find the gaps in the existing literary works. In most cases, the marker based segmentation is best because it marks the part of segment. The working of this proposed algorithm is checked by optimization of the part that is still an area of research.
\end{abstract}

\section{Keywords: ..}

\section{Introduction}

Segmentation of an image can be defined as the division or separation of the image into regions of similar attributes [1]. Major goal of segmentation includes simple replacement of an image into something more meaningful and simpler to analyze. Typically, it is used to differentiate objects in images including lines, curves etc. from image background. Basic attribute for segmentation of color image is monochrome image and color component. Some quality or computed property are similar to each of the pixels in a region. Segmentation procedural goals include simplification of image without discarding original features of selected image [2].

In this paper, theory of marker- controlled watershed transform is discussed and algorithm is applied for few test images. To improve the quality of watershed algorithm and to overwhelm the drawbacks created due to over segmentation; different markercontrolled approaches are elaborated in this paper. A key process in watershed algorithm is the construction of operations among 
neighboring regions for that purpose morphological operations are most valuable.

This process consists of three phases. The first phase is the image pre-processing. Irrelevant information from the image is removed from the first stage. Initial object discrimination becomes the second stage. Groups are entirely isolated into objects with similar attributes. In the third stage, i.e. object boundary cleanup, object boundaries are reduced to single-pixel widths. In the past years, image segmentation have been developed by several popular methods. "Segmentation of an image is the division or separation of the image into parts of similar attribute"[3].

This paper is divided into multiple sections. Section I contains basic introduction of research problem statement, section II will explain the related work, Section III explains research methodology/ basic implementation of solution to problem and Section IV will conclude the research findings and future work will be explained.

\section{Watershed Transformation:}

The watershed transform has captivating properties that make it valuable for different image division applications. In the light of scientific morphology, this transformation is considered as a capable apparatus for image division. Isolating objects can be changed into a watershed. This transformation considers gradient magnitude of image as a topographic layer. Region wise boundaries represent pixels having highest Gradient Magnitude Intensities (GMI's) related with watershed lines. Water placed on any pixel enclosed by a common watershed line flows downhill to a common
Local Intensity Minimum (LIM). A segment is represented when pixels draining to a common minimum form a catch basin. This procedure closes when the water level has achieved the most.

\section{Marker Controlled Watershed Segmentation:}

The issue of over segmentation is experienced by watershed segmentation. The perfect answer for this issue is markers-based segmentation. A marker consists of segment having a place with an image. Determination of marker involves two stages. Image is preprocessed and characterized to select a marker. A typical technique for marker determination can be a morphological edge administrator. It can be accomplished by a morphologic remaking with recursive disintegration (erosion) from the regional minima.

This strategy is derived from the way that segmentation was the expensive number of potential minima. Because of their little size, the majority of these minima can be disposed. Two techniques could be utilized by limiting the impact of little spatial points of interest. With the smoothening channel, image can be separated e.g. Gaussian channel. Notwithstanding, morphological operations can accomplish better outcomes and are utilized as a part of this paper. Finally, in the wake of making the marker picture, the watershed segmentation of the binary image demonstrating the watershed lines can be super forced on the original image.

Following algorithm explains the steps for Marker-controlled watershed segmentation. 
Computation of Segmentation Function-at this step an image is placed whose darker regions act as objects for segmentation.

Figuring Foreground Markers -that means these are connected pieces of pixels within each object.

Compute Background Markers - in this phase pixels are detected that are not the part of any object.

Modification of Segmentation Function -modification ensures that it has only minima at foreground \& background marker locations.

Compute Watershed Transform - this phase also ensures modification of segmentation function [4].

\section{Morphological Operation}

"Morphological image processing is a collection of non-linear operations related to the shape or morphology of features in an image" [5].

Morphological operations utilize morphological organizing component with a specific end goal to quantify and clean relating state of a picture. To achieve goal of investigation diminish picture information and to keep essential shape character morphological operations are needed.

\section{Dilation:}

Dilation expands the original image. It can be used for growing features, filling holes and gaps. Dilation is a technique, which is used for mathematical morphology to enhance or clear the image.

\section{Mathematical representation includes:}

A dilation of an image $\mathrm{X}$ by the structure element $\mathrm{Y}$ is given by the set operation.

$$
X \oplus Y=\{(p+q) \mid p \in X, q \in Y\}
$$

Due to dilation, image size increases and this will also affect the boundaries of foreground pixels. This also causes the area to grow and the holes present in that region will gradually become smaller.

\section{Erosion:}

"An erosion (usually represented by $\ominus$ ) of an image $\mathrm{X}$ by the structure element $\mathrm{Y}$ is given by the set operation"

Mathematically denoted by

$X \bigotimes Y=\{p \in Z 2 \mid(p+q) \in X$, for every $q \in Y\}$

During Erosion, objects start to shrink or may become thin in their respective size this is because it erodes away the foreground boundaries. It will result pixels to shrink in size and holes to become larger.

\section{Closing:}

Closing involves an enlargement took after by disintegration and can be utilized to fill in gaps and little holes[6].

Closing operation is a dilation followed by an erosion:

$$
\mathrm{X} \cdot \mathrm{Y}=(\mathrm{X} \oplus \mathrm{Y}) \ominus \mathrm{Y}
$$

Holes in the foreground that are smaller than $\mathrm{H}$ will be fill.

\section{Opening:}

Opening comprises of a disintegration took after by an enlargement and is utilized to kill 
all pixels in districts that are excessively minute, making it impossible to incorporate the organizing component [7].

Opening operation is an erosion followed by a dilation:

$$
\mathrm{X} \circ \mathrm{Y}=(\mathrm{X} \ominus \mathrm{Y}) \oplus \mathrm{Y}
$$

A frequent call is made against structuring element because image is responsible to question for looking small objects to pull out of image. Lost background remains part of structures that are smaller than $\mathrm{H}$ structure. Eventually, elements will disappear.

\section{Related work}

The least difficult technique for picture division is the Threshold strategy, which in view of an edge esteem. This esteem transforms a dim scale picture into double picture[8].

The primary issue of watershed change is the over division. This is because of the calculation tendency that is exposed to any neighborhood in picture. It also tends to define watershed transform lines where each local minimum gives rise to a region. As a proposed solution for this problem, multiple powerful tools are listed in literature[9].

Performance of image segmentation is satisfactory. It is comparable to other methods that are mostly in use for practicing and developing different techniques. Multiple factors including intensity, texture, and image content are thoroughly dependent in segmentation result. Therefore, this shows that segmentation methods did not work well for one particular image neither for single segmentation application that applies to all type of images [10].

Over segmentation arises due to noise and quantization error problem. Gradient of actual picture is destructing the impact of local minima from noise while causing quantization mistake in result. After that watershed, transformation is applied on gradient image [11].

As compared to the impact of last local minima from noise or quantization blunder the gradient of the actual picture is figured as a pre-processing. The watershed change is connected on the gradient of image afterwards [12].

Morphology is said to be the distribution of image handling activities that will handle images on their shapes premise. In its operation, constructing element used to input image to create a same size output image. Amid morphological task, worth of each pixel made in view of a correlation among relating pixel in input image and alongside its neighboring images. In order to process image depending upon the number of pixels added or removed from objects, the size and shape of structuring elements are used [13].

As per above-mentioned definitions of segmentation, it is clear that the majority of thrust is based on determination of appropriate homogeneity measure that can distinguish objects from each other. Some other examples of measure could be spectral, shape, texture and contexture. Major part of the techniques are connected to remote detecting images included from various different spaces like 
color image segmentation and medical image segmentation and so forth. They intended to work well because of the underlying matching principle. It has extended the application of monochrome (single band) segmentation method, which was originally used on medical imagery, to color image segmentation (three bands)[14].

\section{Implementation and Results}

This research has implemented the marker-based watershed algorithm. Different steps of morphological operations with thresholding process are implemented using MATLAB on multiple images. Finally, the satisfactory results are obtained. However, results are represented in shown below images.

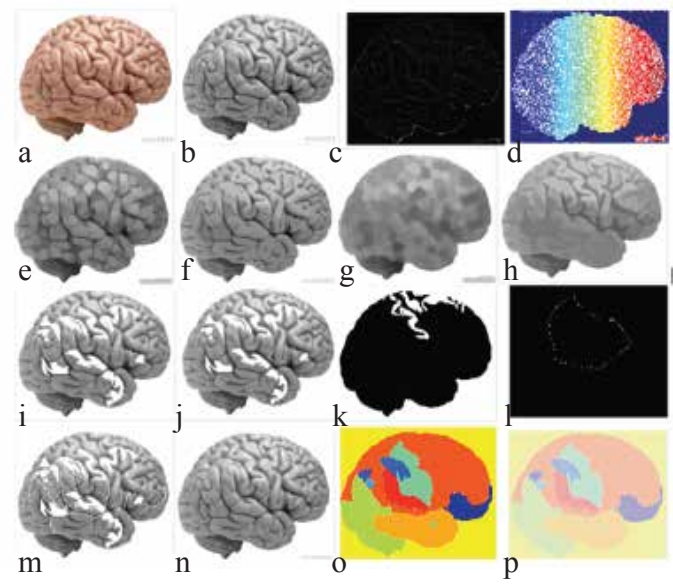

In Figure (1-a) and (1-b)shows the original reference and grayscale image. In figure (1-c) Sobel filter is applied to take gradient magnitude of the image. In figure (1-d) is watershed transform of gradient magnitude image. In figure (1-e) is using morphological operation (opening). In figure (1-f) use reconstruct function. In figure $(1-g, h)$ some morphological operations are applied. In figure (1-i) regional maxima are taken. In figure (1-j) take regional maxima superimposed on original image. In figure (1-k) modified regional maxima (thresholding) is considered. In figure (1-1) ridgelines are washed. In figure (1-m) Markers and object boundaries superimposed on original image. In figure (1-n) colored watershed label matrix is discussed. In figure (1-o) LRGB superimposed transparently on original image.

\section{Summary and conclusion:}

Marker based solve the problem of over segmentation related to watershed model. This technique has shown quite comparable results for all the pixels. However, this technique is only able to manage touched or small overlap inside the image though successful for a huge overlap. Similarly, more research in this discipline might also improve the segmentation accuracy and provide higher consequences.

\section{References}

[1] M. C. J. Christ, R. M. S. Parvathi, M. C. J. Christ, and R. M. S. Parvathi, "Segmentation of Medical Image using Clustering and Watershed Algorithms," Am. J. Appl. Sci., vol. 8, no. 12, pp. 1349-1352, Oct. 2011.

[2] R. S and A. Khan M., "Bio-medical Image Segmentation Using Marker Controlled Watershed Algorithm: A Case Study," Int. J. Res. Eng. Technol., vol. 03, pp. 26-30, May 2014.

[3] B. Sowmya and S. Bhattacharya, "Colour Image Segmentation Using 
Fuzzy Clustering Techniques," in 2005 Annual IEEE India Conference Indicon, 2005, pp. 41-45.

[4] K. Anuradha and K. Sankaranarayanan, "Detection of oral tumor based on marker-controlled watershed algorithm," Int. J. Comput. Appl., vol. 52, no. 2, 2012.

[5] O. Marques, "Morphological Image Processing," in Practical Image and Video Processing Using MATLAB®, John Wiley \& Sons, Inc., 2011, pp. 299-334.

[6] R. Srisha and A. Khan, "Morphological Operations for Image Processing: Understanding and its Applications," presented at the NCVSComs-13, Vignan's University, 2013.

[7] S. Rani, D. Bansal, and B. Kaur, "Detection of edges using mathematical morphological operators," Open Trans. Inf. Process. vol. 1, no. 1, pp. 17-26, 2014.

[8] A. Verma, "The Marker-Based Watershed Segmentation- A Review," Int. J. Eng. Innov. Technol. IJEIT, vol. 3, no. 3, pp. 171-174, 2013.

[9] A. EL Allaoui and M. Nasri, "Medical Image Segmentation By Markercontrolled Watershed and Mathematical Morphology," Int. J. Multimed. Its Appl., vol. 4, no. 3, p. 9, 2012.

[10] Process Dynamics and Control, 4th
Edition. .

[11] F. K. Siddiqui and V. Richhariya, "An Efficient Image Segmentation Approach through Enhanced Watershed Algorithm," Digit. Image Process. vol. 5, no. 3, pp. 132-138, 2013.

[12] S. F. R. Vineet, "An Efficient Image Segmentation Approach through Enhanced Watershed Algorithm," Comput. Eng. Intell. Syst., vol. 4, no. 6, pp. 1-8, 2013.

[13] H. Tulsani, S. Saxena, and N. Yadav, "Segmentation using morphological watershed transformation for counting blood cells," IJCAIT, vol. 2, no. 3, pp. 28-36, 2013.

[14] V. Dey, Y. Zhang, and M. Zhong, "A review on image segmentation techniques with remote sensing perspective," ISPRS TC VII Symp. - 100 Years ISPRS, vol. 38, Jan. 2010. 